\title{
A Statistical Analysis of Subjective and Objective Methods of Evaluating Fabric Handle
}

\author{
Part 1: Analysis of Subjective Assessments \\ By A. E. Stearn*, R. L. D'Arcy*, R. Postle**+ and T. J. Mahar**+ \\ ${ }^{+}$Members, TMSJ \\ *CSIRO Division of Textile Physics, 338 Blaxland Road, Ryde, NSW, 2112 Australia \\ **University of New South Wales, School of Textile Technology, \\ P. O. Box 1, Kensington, NSW, 2033 Australia
}

Based on the Journal of the Textile Machinery Society of Japan, Transactions, Vol. 38, No. 8, T157-T168 (1985-8)

\begin{abstract}
A survey of the fabric handle preferences of panels of expert judges drawn from the textile and clothing industries of Japan, Australia, New Zealand and India has been undertaken. These data have been analysed using two statistical techniques, the first to select the most reliable judges and the second to analyse national judging characteristics.

The technique for selecting the most reliable judges employs a linear model in which each judge's performance is tested against an orthogonal basis of fabric judgment calculated from the assessments of the remaining judges. In an iterative process, the judge whose hadle assessments are least explained by all the other expert judges is then sequentially eliminated until 8 of the original 53 judges remain. This final group was comprised of 4 judges from Japan, 3 from Australia and 1 from India. The results for the New Zealand judges indicate that they include some different characteristics when assessing fabric handle.

The results from a principal component analysis of variance that although a single factor can account for a substantial amount of the observed variability in the data, additional factors are clearly implicated. The three most significant of these additional factors can be related to distinctive national handle preferences by the Japanese, New Zealand and Australian judging panels.
\end{abstract}

\section{Introduction}

The concept of fabric handle has long been used in the textile and clothing industries as a description of fabric quality and prospective performance in a particular application. The subjective assessment of fabric handle has traditionally been made by various experts within the textile and clothing industries. As early as 1930, attempts were made to obtain objective measures of fabric handle and Peirce ${ }^{[1]}$ reported the isolation of various sensations experienced when judging fabric handle and devised measures for these properties, notably fabric stiffness and hardness. The question of fabric handle measurement has received considerable exposure in the literature over the past 50 years with thse techniques of handle assessment being the subject of a recent review article by Ellis and Garnsworthy ${ }^{[2]}$. In particular, extensive work has been reported by Kawabata and $\mathrm{Niwa}^{[3,4]}$ who separated the various primary components of fabric handle assessments for a very wide range of men's suiting materials. These assessments were made by experts from the Japanese textile industry.

A survey has been undertaken of the fabric handle preferences of panels of expert judges from the textile and clothing industries of Japan, Australia, New Zealand and India. An analysis of these assessments which involve 214 men's suiting fabrics has previously been reported ${ }^{[5]}$. This paper provides some results from a more detailed statistical analysis of subjective handle assessments by panels of judges from the four countries mentioned. A later paper in this series will consider the detailed analysis of the objective measurement of fabric handle on technique outlines by Kawabata et al. ${ }^{[3,4]}$

\section{Method}

Data concerning the fabric handle preferences of $\mathbf{5 3}$ judges constituting panels from Japan (8 judges), Asutral- 
ia (18 judges), New Zealand (13 judges), and India (14 judges) have been collected. Each judge was asked to rate the Total Hand Value (THV) of 214 men's suiting fabrics according to the scale shown in Table 1. Judges were

Table 1 Rating scale for fabric handle assessments

\begin{tabular}{c|l}
\hline \hline THV & Evaluation \\
\hline 5 & excellent \\
4 & good \\
3 & average \\
2 & below average \\
1 & poor \\
0 & out of use \\
\hline
\end{tabular}

instructed to rate handle of each fabric according to his or her experience while ignoring the effects of colour and pattern. In this paper, the data have been analysed by two statistical techniques to select the most reliable judges and to expose differences between the various national judging panels.

\subsection{Assessment of individual judges}

This technique allows for accommodation of national preferences whilst still comparing all judges individually. The basis for selecting the most reliable judges is a linear model, in which each judge's performance is tested for a least-squares fit with a linear combination of the other judges' assessments.

The data from all the judges were normalized and, in addition, the data from all, except the judge being tested, were orthogonalised. The sum of the squares of the correlations between the scores by the judge being tested and the columns of the normalized orthogonal basis was used as a figure of merit, to define how the findings of the judge being tested could be explained by the other judges, see Yassa $^{[6]}$. The judge giving the smallest value was rejected, and the procedure was iterated until only two judges remained.

\section{A. Theoretical basis}

To understand the linear model for the judging process, it is assumed that any fabric can be fully described by a finite number of physical attributes. However, the exact number and nature of all these descriptors is not known.

When a fabric is subjectively assessed, the judge responds to a selection of the fabric attributes and either consciously or unconsciously, bases his assessment on some function of the selected attributes. Consider ' $n$ ' $\times$ ' $m$ ' matrix, $A$, in which the individual values for each of ' $m$ ' fabric attributes, arranged as columns, are tubulated for each of ' $n$ ' samples (or fabrics) arranged as rows.

Individual judges may well have different responses to any given set of parameters and this variation can be expressed as a weighting factor for each parameter. A second matrix, $J$, can be used to store these ' $m$ ' weighting factors from each of ' $p$ ' judges, or observers.

Any data matix, $D$, containing, for example, the Total Hand Value assessments of each observer for all samples can be expressed as the matrix product of the matrices $A$ and $J$, i.e.

$$
D=A . J
$$

It should be noted that the rank of the matrix $A$ limits the rank of $D$, so that irrespective of the number of observers involved there is a constant number of significant factors involved.

The handle data from any single judge can be isolated by selecting the appropriate column vector from the matrix $D$. If the selected column vector is $H$, any of the remaining columns of the original matrix $D$ may be expressed as a linear combination of the columns of a new orthonormal matrix, $U$. This matrix, is composed of orthogonal (i.e. completely independent) columns, which constitute a vector space, from which the remaining judge's data may be recontituted.

The selected vector $(H)$, obtaining one judge's assessments, may be decomposed into two components, " $h$ " and " $E$ ", i.e.,

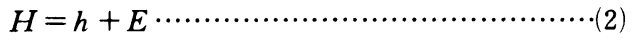

The component $h$ is comprised of that amount of the judge's assessments which are explainable in terms of the other judges' assessments. This component $h$ can be described by terms from the basic orthogonal matrix, $U$, the second component, $E$, represents the amount of the judge's assessment which is unique to that judge. It may be random noise in the judge's assessments, or influences of one or more fabric parameters which only this judge assesses. Because this component, $E$, is orthogonal to the basic matrix, $U$, it must also be orthogonal to the column vector $h$.

If the vector, $H$, is normalized (sum of squares of its elements is unity)

$$
\Sigma\left(H_{i}\right)^{2}=\left(\left(h_{i}\right)+E_{i}\right)^{2}=1
$$

Since $E$ and $h$ are orthogonal,

$$
\Sigma\left(h_{i}\right)^{2}+\left(E_{i}\right)^{2}=1
$$

Suppose now we have found the optimum vector $R$ from which we may calculate $h$ from $U$. Then $h=U R$, substituting this in equation 2 we have

$$
H=U R+E \cdots \cdots \cdots \cdots \cdots \cdots \cdots \cdots \cdots \cdots \cdots \cdots \cdots \cdots \cdots \cdots \cdots \cdots(4)
$$

Premultiplying each term by $U^{T}$ we have

$$
U^{T} H=R+U^{T} E=R \cdots \cdots \cdots \cdots \cdots \cdots \cdots \cdots \cdots(5)
$$

since $U^{T} E=0$ because $E$ is orthogonal to $U$ and $U^{T} U=I . R$ is simply the vector of correlations of $H$ with $U$. Now $E_{i}{ }^{2}$ $=E^{T} E$, so

$$
\begin{aligned}
E^{T} E & =(H-U R)^{T}(H-U R) \\
& =H^{T} H-H^{T} U R-R^{T} U^{T} H+R^{R T} V^{T} U R \\
& =H^{T} H+R^{T} R
\end{aligned}
$$

since $\Sigma H^{T} U$ and its transpose are zero. In equation ${ }^{[3]}$ then $h_{i}{ }^{2}$ becomes $\Sigma R_{i}{ }^{2}$ or

$$
\Sigma R_{i}^{2}=1-E_{i}^{2}
$$

This sum of squares of correlations, which for a normalized matrix, lies between 0 and $1\left(0 \leq R_{i}{ }^{2} \leq 1\right)$, can be regarded as the proportion of the selected judge's find- 
ings that are accounted for by the findings of the remainder of the judging panel.

\subsection{Characteristics of national judging panels}

In order to investigate the causes of disagreement between judges, a Principal Component Factor Analysis ${ }^{[7]}$ (P.C.A.) was performed on the total hand value data from the 53 judges for 214 fabrics. In this analysis the data matrix, containing the THV assessments of the judging panel, is decomposed into a matrix of orthogonal factors and a matrix of individual judges weightings of each factor. The original data matrix can then be reconstructed from a linear combination of the orthogonal factors, which may be arranged in order of the amount of variability in the judges assessments which they can account for. The decomposition of the data matrix also produces a weighting matrix in which each judge's individual preference for each of the independent factors is tabulated. Obviously, judges who assess the same orthogonal factors, even though they may utilise a different set weightings, will correlate more highly with each other than judges who assess different factors all together. The major advantage of a P.C.A. is that it enables the variation of a large number of individual judge's estimates to be expressed, with an accuracy depending on the number of factors, by a much smaller number of independent factors.

Although the P.C.A. can accurately determine the number of orthogonal factors needed to represent the judge's assessments, it is not a good technique to enable the identification of judges with similar judging patterns. However, for any selected number of factors, deemed to be most significant in explaining the observed variance in the original data matrix, it is possible to carry out the decomposition of that data matrix to produce a set of 'varimaxed' factors ${ }^{[8]}$ which, whilst still being orthogonal, have been rotated in such a way that the correlations between the varimaxed factor scores and the judges original scores are either maximised or minimised. This helps to identify those judges who assess individual factors similarly.

\section{Results and Discussion}

\subsection{Variation of judges' assessments}

The means and standard deviation of the national panels of judges are given in Table 2. It can be seen from these

Table 2 Means and standard deviations of the fabric handle assessments for the four national panels of judges

\begin{tabular}{l|c|c}
\hline National panel & Mean & $\begin{array}{c}\text { Standard } \\
\text { deviation }\end{array}$ \\
\hline Japan & 3.15 & 0.89 \\
India & 3.07 & 1.19 \\
Australia & 3.06 & 1.15 \\
New Zealand & 3.05 & 1.09 \\
\hline
\end{tabular}

figures, that judges of country rated the fabrics to a similar mean and standard deviation. The means and standard deviations of each individual judge have been included in the appendix.

\subsection{Sequential elimination of unsupported judges}

It would be expected that if each of the 53 judges was assessing the handle of the fabrics by precisely the same definition of fabric handle, then the assessments of each judge should be almost totally predictable by the remaining 52 judges. However, this examination exhibited large variation in the degree to which the results of individual judges were accounted for by the assessments of the remainder of the panel. Figure 1 shows the distribution of the number of judges with a nominated proportion $\left(\Sigma r_{i}{ }^{2}\right)$ of their fabric handle assessments being calculable from the assessments of the remaining 52 judges.

The amount of variance explained by this technique ranges from a minimum of $40 \%$ for three of the judges to a maximum of $90 \%$ for one judge. This implies that there are three judges of fabric who each have at least $60 \%$ of the variability in their assessments unsupported by 52 of their peers. Conversely, one judge has $90 \%$ of the variability of his assessments supported by the assessments of the other 52 judges. The average level of explanation of the handle assessments of each judge in terms of the other 52 judges is (approx.) $70 \%$.

This relatively large variability in fabric assessment may be caused by the fact that either, judges are assessing fabric handle with different fundamental concepts, or that there is a high level of randomness in the assessment of the

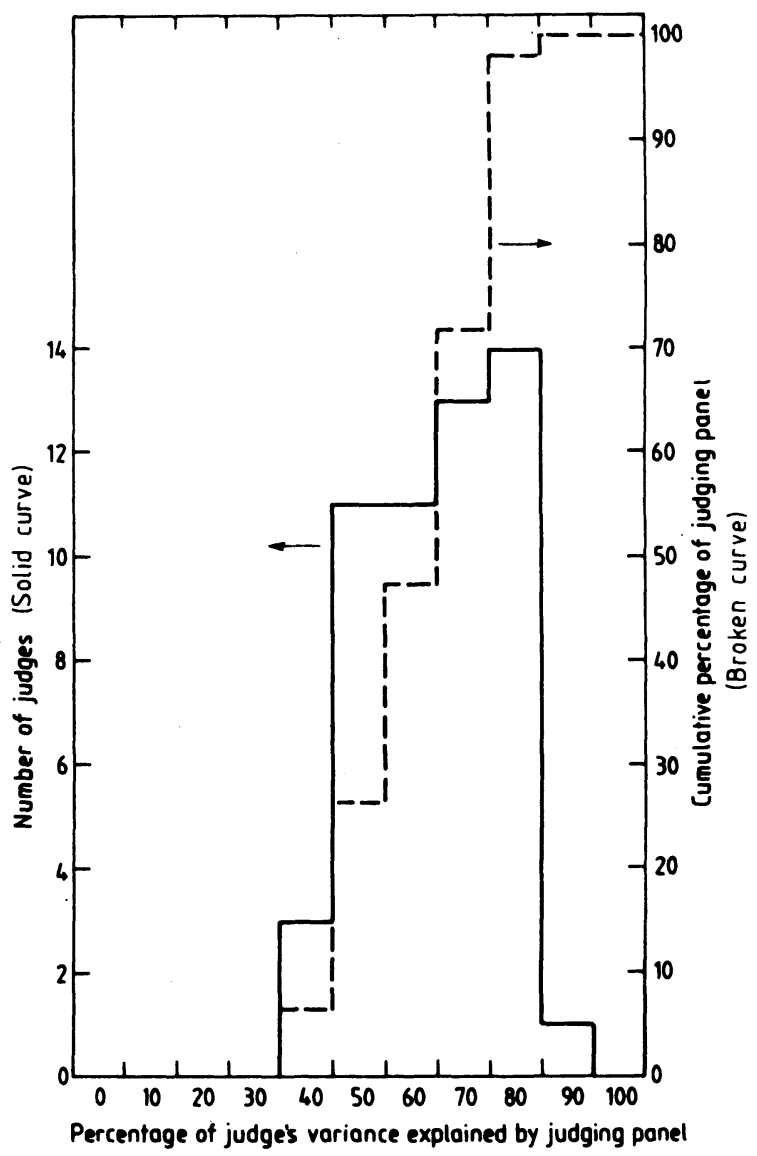

Fig. I Showing number of judges and cumulative percentage of full judging panel vs. fraction of each judge's actual assessment calculated from the assessment of the 52 remaining judges in the panel. 


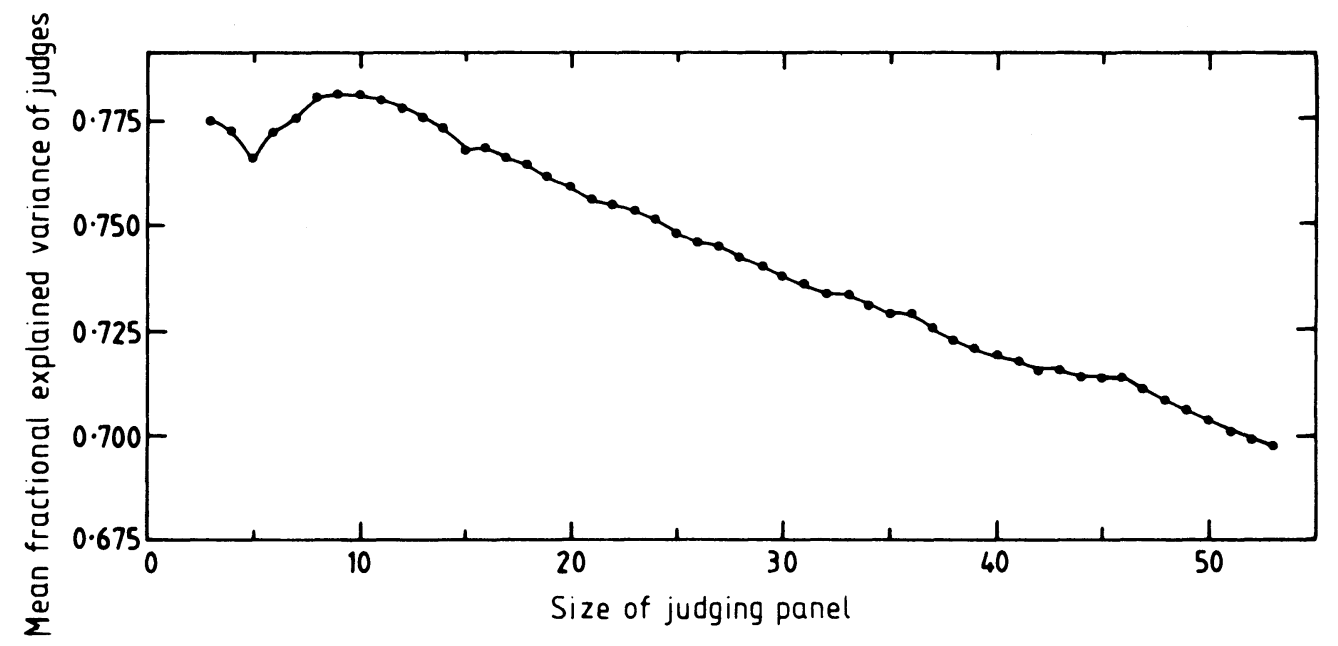

Fig. 2 Mean fractional explanation judges' THV assessments by judging panel vs. size of judging panel (as judges are selectively eliminated).

majority of the judges. Since the judge with the lowest explained variance appears to be assessing some fabric features which are being completely ignored by his peers, his ratings can be considered the most unreliable of all the 53 judges. When this is eliminated from the judging panel, the average $\Sigma r_{i}{ }^{2}$ value of all members of the judging panel is likely to be modified. Such an elimination process can be continued until there is a much smaller panel of selfconsistent judges remaining. Figure 2, shows the mean fractional predictability of judge's assessments plotted against the number of judges remaining in the judging panel as the less predictable judges are eliminated. This figure clearly demonstrates that the average explanation of each judge's fabric handle assessments in terms of the assessments, of his fellow judges, generally improves as the size of the judging panel is selectively reduced.

The presence of minimam in the average level of explanation, when both 5 and 15 judges are in the panel is significant in that it implies that these remaining judges have lost some support for their assessments by the elimination of judges 5,6 and 7 and judge 15 respectively. It appears that there are 2 distinct groups of judges remaining after the elimination of 45 of the original 53 judges. Judges 5, 6 and 7 , comprise a relatively selfconsistent sub-set of the final 8 remaining judges who come from Japan (judges 1, 2, 3, 4), Australia (judges 5, 6 , 7 ) and India (judge 8). The idea that there may be subtle but distinct national preferences in the handle assessments of these judges is reinforced by consideration of Fig. 3. This figure demonstrates the national compositon of the judging panel as each judge is successively removed from the panel.

It can be seen that the assessments of the New Zealand judges lack support, both from within their own panel and from the wider international panel. This result seems to indicate either the possibility of a distinctly New Zealand concept of handle, or a relatively high degree of randomness within the New Zealand panel. On the other hand, the smaller Japanese panel had only one of its judges in first 30 who were eliminated, and thus maintained a high proportion of the more consistent members of the judging panel. The Indian panel shows

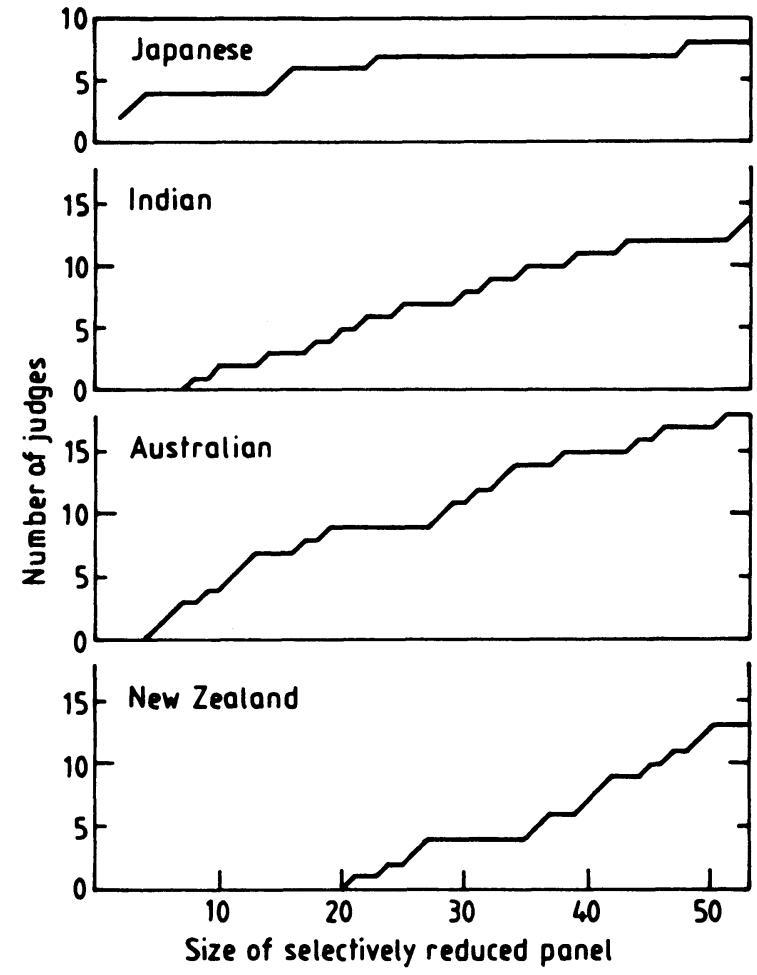

Fig. 3 Composition of judging panel unsupported judges are eliminated.

a reasonably constant rate of rejection of its judges over the middle of the range of numbers of judges remaining. This indicates that these judges do not strongly support a national characteristic preference. The Australian panel shows an inital constant rate of rejection of judges until there are 9 Australians among the final 27 judges. After only losing another 2 in the next 14 to be eliminated, the final 7 Australian judges were then quickly eliminated. This result indicates that the sub-group of the most consistent Australian judges has a characteristic fabric handle preference which is not shared by the last 4 remaining Japanese expert judges. 


\subsection{Factor Analyses}

The possibility of national judging panels for fabric handle having distinctive national preferences in fabric handle assessments was further investigated using the factor analysis technique of Principal Component Analysis.

The results from a principal component analysis of variance given in Fig. 4 show that although a single factor

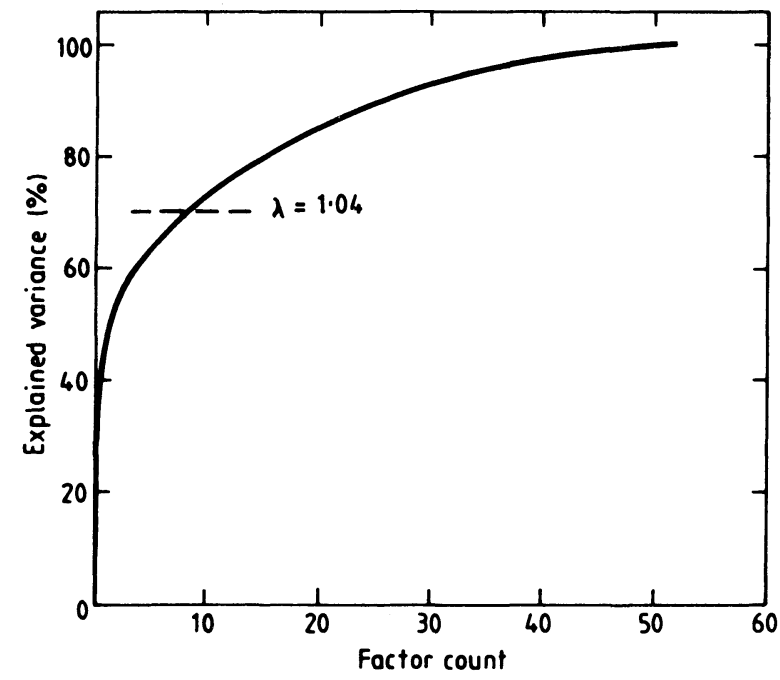

Fig. 4 Explanation of observed variance vs. factor count.

can account for a substantial amount of the observed variability in the data, additional factors are clearly implicated. Consequently, the assessment of handle may be considered as a linear model, in which, judges rank fabrics by a number of properties which are given different weightings by individual judges.

The first 9 factors, which collectively account for approximately $71 \%$ of the observed variance, were arbitrarily chosen for investigation on the basis that the eigenvalue for the ninth factor was approximately equal to one. When these 9 factors were varimaxed, it was found that the correlations between individual judges with each of the varimaxed factors tended to be polarized. A correlation of 0.3 was arbitrarily selected as the lower level at which an individual judge would be deemed to regard a factor as being significant. By counting the judges who considered each factor as being significant, it was found that distinct national judging characteristics emerged.
Table 3 summarises these results. Four factors were each able to explain more than $5 \%$ of the observed variance, whilst the most important factor accounted for some $35 \%$ of the observed variance. This factor (factor No. 1) was recognized and weighted heavily by most of the judges indicating a strong level of overall agreement about fabric handle among the national panels. The factor which has the next highest level of variance explained, a little over $10 \%$, was highy related to all of the Japanese panel, 6 of the Australian judges and 2 of the Indian panel. This

Appendix

Means and standard deviations of the fabric handle assessments of 214 winterweight men's suiting fabrics from Japan, India, Australia, and New Zealand.

\begin{tabular}{cccccc}
\hline \multicolumn{6}{c}{ Japanese judges } \\
No. & Mean & SD & No. & Mean & SD \\
1 & 3.26 & 1.11 & 5 & 3.10 & 0.92 \\
2 & 3.12 & 0.97 & 6 & 3.41 & 0.61 \\
3 & 3.08 & 0.88 & 7 & 3.03 & 1.03 \\
4 & 3.10 & 0.73 & 8 & 3.09 & 0.87 \\
Indian judges & & & & \\
1 & 2.79 & 0.60 & 8 & 2.64 & 1.12 \\
2 & 3.50 & 0.49 & 9 & 3.045 & 0.61 \\
3 & 2.72 & 1.51 & 10 & 1.80 & 1.82 \\
4 & 3.23 & 0.99 & 11 & 2.83 & 1.42 \\
5 & 2.72 & 0.99 & 12 & 3.66 & 0.85 \\
6 & 3.46 & 0.79 & 13 & 3.34 & 0.96 \\
7 & 3.75 & 0.95 & 14 & 3.440 & 1.19 \\
Australian judges & & & \\
1 & 3.07 & 1.26 & 10 & 3.51 & 1.05 \\
2 & 2.26 & 1.25 & 11 & 3.30 & 0.83 \\
3 & 2.61 & 1.33 & 12 & 3.75 & 0.96 \\
4 & 2.27 & 1.05 & 13 & 3.39 & 1.00 \\
5 & 3.17 & 1.56 & 14 & 3.869 & 0.85 \\
6 & 3.44 & 0.82 & 15 & 2.89 & 1.51 \\
7 & 2.52 & 1.31 & 17 & 2.77 & 1.18 \\
8 & 2.71 & 1.37 & 18 & 3.69 & 0.89 \\
9 & 2.62 & 1.41 & & & \\
New Zealand judges & & & \\
1 & 3.06 & 1.51 & 8 & 3.44 & 1.12 \\
2 & 2.94 & 0.87 & 9 & 2.60 & 0.97 \\
3 & 2.00 & 1.45 & 10 & 3.26 & 1.05 \\
4 & 2.79 & 1.13 & 11 & 3.22 & 0.88 \\
5 & 3.18 & 1.19 & 12 & 2.80 & 1.18 \\
7 & 3.71 & 1.10 & 13 & 3.000 & 0.87 \\
\hline \multicolumn{7}{l}{} & & & & \\
\hline
\end{tabular}

Table 3 Showing \% of National judging panels significantly influenced by individual independent varimaxed factors assessed in the judgement of Total Hand Value.

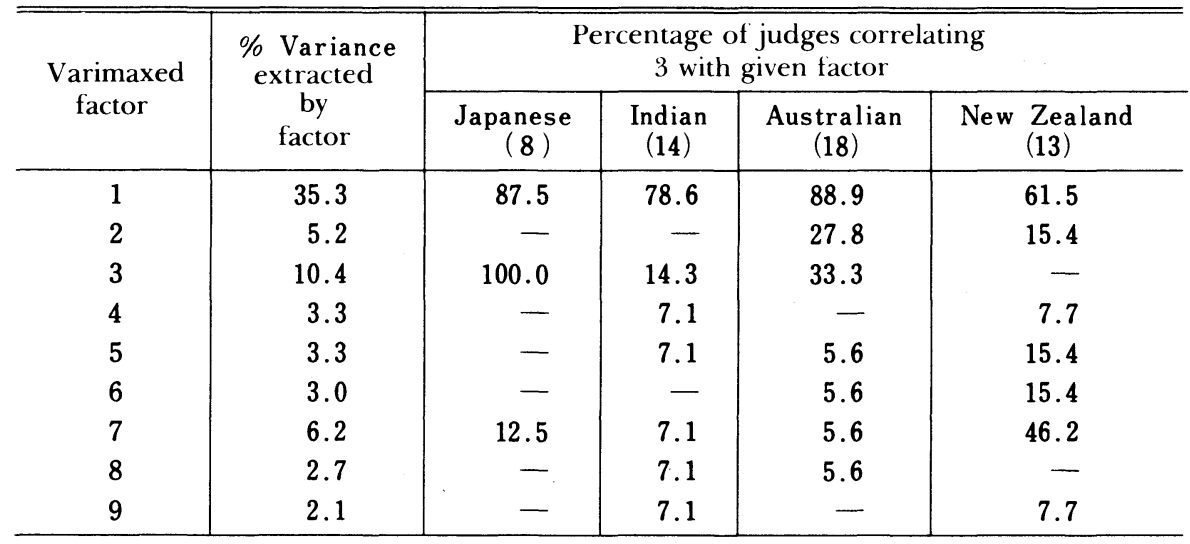


second factor therefore denotes a strong Japanese character which is alos evident in a significant proportion of the Australian panel of judges. The third factor, which accounts for a further $6 \%$ of the variance, was supported to a significant extent only by the New Zealand panel. The fourth factor which explains $5.2 \%$ of the variance has a significant relationship with the Australian panel, 5 of the 18 Australian judges having a correlation with this factor of greater than 0.3 , and a similar relationship with 2 of the 13 New Zealand judges. The remaining five factors, which were each only able to account for less than $3.5 \%$ of the observed variance, failed to show any strong tendency to be associated preferentially with any one nationality of judging panel. Rather they are related to two or three individual judges from different countries.

\section{Conclusion}

The statistical procedure for the sequential elimination of the judge whose assessments are least explained by his peers has shown that relatively self-consistent sub-groups of 8 and 16 of the original 53 judges can be obtained. Each of these sub-groups contain judges from Japan, Australia and India. The results for the New Zealand judges indicate that they may include some different characteristics when assessing fabric handle. It should be emphasized that the sub-group of 8 expert judges can be further sub-divided along national lines. This final division indicates that, despite a strong overall agreement between the national judging panels, there may be subtle but distinct national preferenses in their handle assessments.

The results of the principal component analysis of variance support the view that there are national preferences in the fabric assessments of the judges from the 4 countries studied. These results indicate that, although a single factor can account for a substantial amount of the observed variability in the data, additional factors show preferences for the various national groups.
This paper represents the first par of a series to analyes underlying factors involved in subjective and objective methods of evaluating fabric handle. The subjective assessments have now been extended to include the U.S.A. and a preliminary analysis of these results has been reported elsewhere (Fabric Handle Equations for Aust., N.Z., India and U.S.A.). The international fabric handle survey is also being carried out in Germany. Part 2 of this series of papers will be concened with a statistical analysis of the objective method of evaluating fabric handle using fabric mechanical and surface property measurements.

\section{References}

[1] Peirce, F. T.; J. Text. Inst., 21, T377 (1930).

[2] Ellis, B. C. and Garnsworthy, R. R.; Text Res. J.,50, 231 (1980).

[3] Kawabata, S.; "The Standardization and Analysis of Hand Evaluation", 2nd Ed. The Textile Machinery Society of Japan (1980).

[4] Kawabata, S. and Niwa, M.; Analysis of Hand Evaluation of Wool Fabrics for Men's Suits using Data of a Thousand Samples and Computation, Proc. Fifth International Wool Textile Research Conference, Aachen, 5, 413 (1975).

[5] Mahar, T. J. and Potle R.; International Fabric Handle Survey, in "Objective Evaluation of Apparel Fabrics", ed. by R. Postle, S. Kawabata and M. Niwa, The Textile Machinery Society of Japan, p. 261 (1983).(Proceedings of the Second Australia-Japan Bilateral Science and Technology Symposium on Objective Evaluation of Apparel Fabrics, Parkville, Victoria, Australia, 24 October-4 November 1983).

[6] Yassa, G.; J. Photogrammetric Engineering,40, No. 8,961 (1974).

[7] Cooley, W. W. and Lohnes, P. R.; "Multivariate Data Analysis”, Wiley, U.S.A. Ch. 4, 5 (1971).

[8] Kaiser, H. F.; Psychometrika, 23, 187 (1958). 